\title{
Comparative study of battery energy storage systems in a micro-grid based on real-time simulation
}

\author{
Karim FELLAH ${ }^{1,2}$, Rosa ABBOU ${ }^{2}$, Mounir KHIAT ${ }^{1}$, Rahiel DJELLOUL ${ }^{1}$ \\ ${ }^{1}$ SCAMRE Laboratory, National Polytechnics School of Oran-Maurice Audin, Algeria \\ ${ }^{2}$ LS2N Laboratory, University of Nantes, France
}

\begin{tabular}{l}
\hline \hline Article Info \\
\hline Article history: \\
Received Dec 23, 2020 \\
Revised May 7, 2021 \\
Accepted May 25, 2021 \\
\hline
\end{tabular}

\section{Keywords:}

Renewable energies

Micro-grid

Power fluctuations

Energy storage system

Batteries

\begin{abstract}
The penetration of the distributed energy resources particularly solar and wind power into the electrical system has been increasing, but the intermittent nature of these resources produces perturbations and instability in the electrical grid. Thus, integrating energy storage system into the power grids is one of the best solutions to improve the reliability with performance of electrical systems; ensure power balance and meet consumer demand. Different technologies of energy storage devices have been used to support the integration of renewable energy resources and contribute to improve either the effectiveness with the management of power operating system during critical situations such as power fluctuations in the electrical grid. The main objective of this work is to test the effectiveness of battery energy storage system in reducing active power fluctuations in presence of a perturbation in a micro-grid. In addition, a comparative study is carried out by comparing the response of different battery technologies which are used to support the electrical grid in order to verify the appropriate battery technology for power systems and particularly for energy management in micro-grids during power fluctuations, also, the evaluation of the behaviour, feasibility, performance and effectiveness of BESS is achieved by using real-time simulation.
\end{abstract}

Copyright $\left({ }_{0} 2019\right.$ Institute of Advanced Engineering and Science. All rights reserved.

\section{Corresponding Author:}

Karim FELLAH,

SCAMRE Laboratory,

National Polytechnics School of Oran-Maurice Audin,

BP 1523 Oran El M'naouer, 31000 Oran, Algeria,

Email: karim.fellah@enp-oran.dz

\section{INTRODUCTION}

The unpredictable power fluctuations with intermittent nature of renewable energies may cause power unbalance which affects micro-grids operation. To reduce these disturbances and maintain balance between power generation and demand, Energy Storage Devices (ESDs) are used as an effective solution. In fact, in the distribution grids, once a fault is detected the distributed generators (DGs) should be disconnected from the grid; the objective of this action is to prevent islanding but this behaviour can cause many issues like: (a) safety problems due to abrupt partially energized grid, (b) perturbation in frequency and voltage control...etc, thus it leads to network instability. However, when the micro-grid is connected to the main grid (on-grid mode), both voltage and frequency are controlled by the utility grid (main grid). Therefore, several grid codes require that renewable energy sources (RESs) should maintain their connection to the electrical grid during disturbances in order to contribute for electrical power system stability. Such assistance is ensured by voltage and frequency regulation through active and reactive power support requirements [1], [2]. To reduce these issues and maintain balance between power generation and demand, energy storage devices (ESDs) are used as an effective solution. Energy storage system (ESS) facilitates RESs integration just by storing or delivering energy through the charge and discharge process. Technically, ESS is classified as high power rating for power quality and energy management applications. Within integration of RESs, ESS can smooth energy intermittency, reduce the reverse flow of power and maintains voltage within limits, store energy with local load and control the ramp rate of power fluctuations [3],[4]. The efficient control of ESS used for voltage support helps to reduce 
power fluctuations through coordinated supply of active and reactive power on a local demand point. Inverters based voltage and current are integrated into each ESD in order to maintain voltage and frequency in any microgrid [5]. Global energy storage capacity amounts to approximately 4.67TWh in 2017 and is predicted to rise to 11.89-15.72TWh in $2030^{1}$. With the development in power electronic technology, battery energy storage system (BESS) has become the focus in energy storage systems. Therefore, its role in power system is very important, such as power smoothing and peak shaving for large Photovoltaic (PV) farms, frequency regulation, etc. Different energy storage technologies are used in power systems, while lithium-ion batteries are the most used as means of energy storage. Other technologies like lead-acid and nickel-cadmium batteries, flywheels, super-capacitors, etc are technically and economically competitive. In addition, there are advantages and drawbacks in each technology which is able to provide sufficiently fast response comparing to the conventional sources of energy. Moreover, the combination of various energy storage technologies such as conventional generators with hybrid and ESS or renewable generator could be the best solution. Some authors have adopted such approach by using the concept of virtual power plant techniques [6].

The main objective of this study is to verify the behavior of BESS and how it manages its state-ofcharge (SoC) when it is connected to the electrical power system, therefore ensuring that its available energy can support the micro-grid during critical situations such as power fluctuations and how quickly BESS can deliver or absorb power in response to any undesirable phenomena in order to guarantee power balance with stability in the whole power system. Furthermore, a comparison between different battery technologies is carried out in order to determine which battery type is more adequate for energy management in micro-grids by comparing the response time of lithium-ion, lead-acid and nickel-cadmium batteries in presence of power fluctuations in the micro-grid. In addition, due to the complexity of the whole micro-grid system, this requires more reliable control to regulate the flow and to improve the quality of power, voltage, and frequency. Also, the greater complexity of such systems, the more tests are required, all with high accuracy than ever. Most of existing research about renewable energies mainly focuses on off-line simulation which has to go through a long research period and low efficiency. However, real-time simulation enables researchers to study the effects of multiple scenarios in near real conditions without risk, prior to deployment in the field. Therefore, the present study is achieved by using real-time simulation platform.

The structure of this paper is organized as follows; an overview about the micro-grid architecture is presented in section 2, and then the BESS operation with energy management system in the micro-grid is presented in section 3. In section 4, a modeling and control of BESS is described. A micro-grid model description with its real-time simulation is shown in section 5 and 6 . The simulation scenario and results discussion are shown in section 7 and finally a conclusion is given in section 8 .

\section{ENERGY MANAGEMENT SYSTEM FOR MICRO GRID SYSTEMS}

\subsection{Micro-grid architecture}

As the current electrical power grids integrate distributed energy resources (DERs) more and more, its structure has been very complex. For optimal operation, micro-grids structure is regarded as an effective solution. Many countries have adopted the context of micro-grids mainly in Europe, United States, Australia, and Japan. A micro-grid is a small power network which is composed of DERs, energy storage system (ESS) and loads. It is capable to operate in two modes: grid-connected mode and in islanded mode to ensure the reliability of power supply to the local community. In the standard conditions, the micro-grid operates in gridconnected mode; it operates in parallel with the main grid, therefore, it exchanges power in order to guarantee a power balance between supply and demand for both micro-grid and the utility grid, which is a very important requirement [1], [2], [7]. Integration of DERs into micro-grids is challenging, and one of the main requirements of this integration is to maintain stability during a disturbance. Power grid disturbance causes many technical and economic problems, but an effective control with energy management system lead to reduce power losses and increase reliability. In addition, due to the intermittent nature of RESs which impacts on micro-grids operation, an ESS is added into the micro-grid to store the excess of generated power or deliver power to the electrical grid in case of deficiency in order to meet load demand [2].

\subsection{Micro-grid Control and Energy Management}

The Frequency control is one of the important problems, it is due uncontrollable loads and RESs intermittency, which results as an imbalance between power supply and demand. As it is shown in Figure 1 taken from the reference [8], a micro-grid control centre supervises the operations of the micro-grid components such as connection and disconnection from the main grid, resynchronization, and consumers, etc. Also, this control centre controls the micro-grid either in grid-connected or in the islanded mode. Also,

${ }^{1}$ http://sdg.iisd.org/news/irena-report-expects-global-electricity-storage-capacity-to-triple-by-2030/ viewed: 18-févr-2020

IJEEI, Vol.9, No. 2, June 2021: 371 - 383 
an efficient energy management of micro-grids is needed for high penetration of RESs. The main objective of the energy management system of the micro-grid is to reduce operating cost, maintenance, and the cost of the imported power from the utility main grid [1].

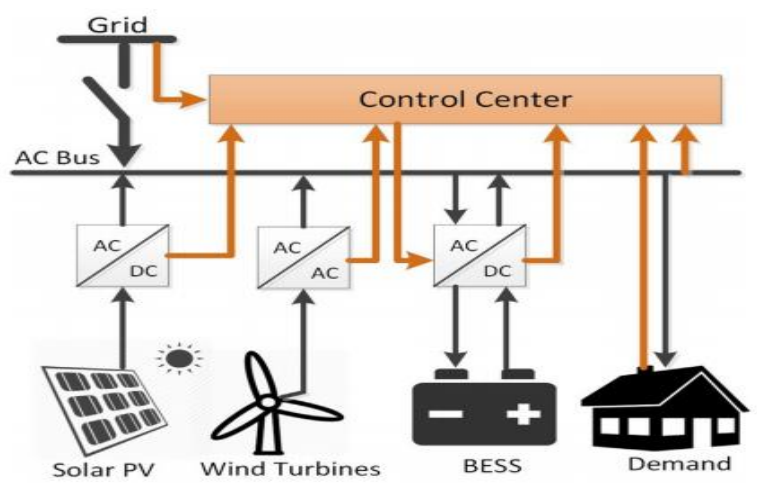

Figure 1. Structure of a micro-grid

\section{BESS OPERATING WITHIN ENERGY MANAGEMENT SYSTEM (EMS)}

Adding energy storage devices to solar panels and wind farms can help to reduce the need for transmission infrastructure by allowing more of the power that is generated by solar panels to be used locally. In some cases, especially in isolated areas, solar with battery systems don't have any connection to a regional grid, only using distribution level infrastructure as it is shownin Figure 1. These types of systems are called "islanded" systems and must generate the power needed to meet local demand. Frequency and voltage are the main parameters which highlight the stability of any power system either for conventional or modern electrical grid. A micro-grid can operate as connected to the main grid (on-grid) or isolated from the main grid (off-grid). For frequency control in isolated micro-grid, an energy storage system is required. In fact, ESDs play role of generators to maintain balance power demand-supply in the entire grid. If initial SoCs of ESDs are different, and in order to avoid the situation when certain ESDs with low SoC stop operating earlier than those with high SoC, the output of each ESD should be regulated according to the initial value of SoC [5]. The active and reactive power injected to the bus by any generator can be expressed as:

$$
\begin{aligned}
& P_{i}=\frac{1}{\sqrt{r_{i}^{2}+x_{i}^{2}}}\left[\left(E V_{i} \cos \theta_{i}-E^{2}\right) r_{i}+E V_{i} \sin \theta_{i} x_{i}\right] \\
& Q_{i}=\frac{1}{\sqrt{r_{i}^{2}+x_{i}^{2}}}\left[\left(E V_{i} \cos \theta_{i}-E^{2}\right) x_{i}-E V_{i} \sin \theta_{i} r_{i}\right]
\end{aligned}
$$

Where $I \in[1, \mathrm{z}], x_{i}$ and $r_{i}$ are respectively the line reactance and resistance; $\theta_{i}$ is power angle; $E$ and $V$ are respectively the amplitudes of voltage and inverter output-voltage on the point of common coupling (PCC) [5]. In order to simplify those relations, we consider that in any power grid, the frequency is related directly to active power variations and voltage is more related to reactive power :

$$
\begin{gathered}
F=f(P) \\
V=f(Q)
\end{gathered}
$$

Where $F$ is the frequency, $P$ is the active power, $V$ is the voltage, and $Q$ is the reactive power. As it is shown in Figure 2, BESS is usually connected to the rest of the system at the point of common coupling (PCC) via power electronic devices such as voltage source converters (VSCs) and bidirectional DC-DC converters [6]. The VSC is used to convert power from DC to AC and The DC-DC converter is used to increase the voltage level from the battery output voltage to the DC-bus voltage. To stabilize and maintain the voltage of the DCbus of the VSC, a buck/boost converter is used despite the exchange of real power to/from the main grid [9]. 


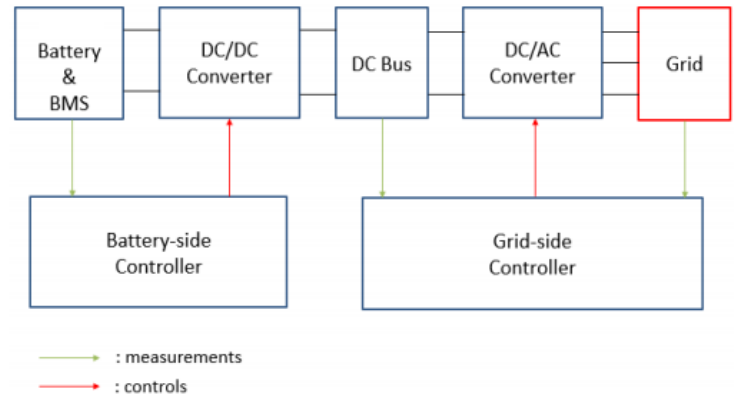

Figure 2. Overall simplified structure of a BESS connected to AC grid [6]

When many BESSs exist in a micro-grid, a coordination is necessary to ensure that the stored energy is flowing among the units in order to avoid deep-discharging and/or over-charging in one of the energy storage units than others [8],[10].

\subsection{State-of-Charge (SoC) in BESS}

The state-of-charge (SoC) is the main parameter to take into consideration for any application using storage system and particularly BESS. This parameter ensures an efficient operation and the security of the batteries. However, the concept of energy management is based on the exact estimation of SoC. Usually, such estimation depends on several factors such as temperature, capacity and internal resistance of the battery [11]. The SoC of a battery is the percentage of its available capacity over its maximum capacity [12]:

$$
\text { SoC }=\frac{\text { available capacity }(\text { Ah })}{\text { maximum available capacity }(\text { Ah })} \times 100 \%
$$

\subsection{Deep-of-Discharge (DoD) in BESS}

Deep-of-Discharge $(D o D)$, is the percentage of the used capacity during a discharge cycle, it is tracked based on $S o C$ shown as [13]:

$$
D o D=S o C_{\text {initial }}-S o C_{\text {final }}
$$

\subsection{Classification of BESS in power grid}

In power system, BESS is divided into two configurations: aggregated BESS and distributed BESS [6]. The performance and applications are determined by these configurations. The aggregated type of BESS is a great energy storage installation, with a dedicated compartment in the micro-grid. Usually, it has very high energy capacity and power capacity. In fact, based on some research, an aggregated ESS is more able and efficient to suppress power fluctuation in the micro-grid than a distributed ESS, this is because the power output in such configuration storage farm is more stable than each separated unit.

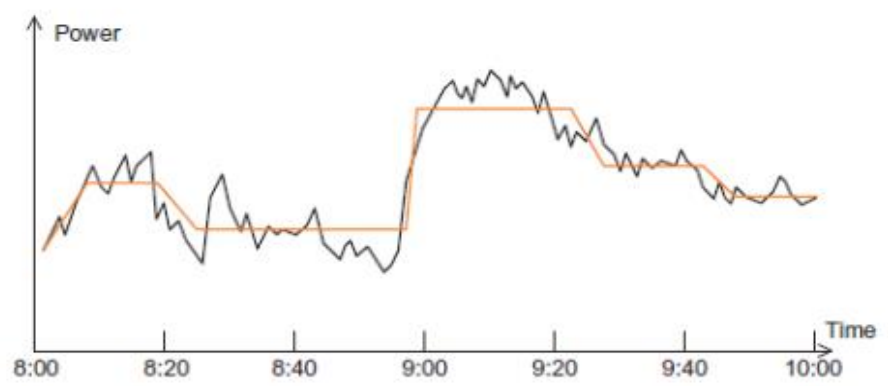

Figure 3. Principle of load consumption: without BESS (black) and with BESS (red) [6]

For distributed BESS, there are two categories; the first one is on the generator side, it helps the DG to smooth the output power. The other type is on the load side which ensures energy management and reduces load variation. Distributed BESS is smaller than aggregated BESS because they handle an individual or a small group of DGs. When used with a renewable generation sources, the distributed BESS is generally connected to the same DC bus, behind the grid-side inverter. In this configuration, the BESS helps to get a stable DC bus voltage by compensating the fluctuations of renewable generation. A BESS is very solicited for energy 
management and stability improvement and economical aspect of a micro-grid. The main energy management functions include "load levelling" and "peak shifting", both of them depend on the variation of demands. Therefore, load levelling focuses on short-term fluctuation, but peak shifting is for the long-term (24h) variation. An example of load levelling is depicted in Figure 3. In this way, load levelling improves the effectiveness and power quality in the micro-grid [6],[14],[15].

\section{BESS MODELING AND CONTROL}

The battery system is a "Battery Pack" which connects multiple cells to get the desired voltage and capacity. BESS control and monitoring is often called as "Battery Management System" (BMS). As functions, the BMS ensures the protection of the cells against harmful operation in terms of voltage, temperature and current to achieve reliable and safe operation, and the balancing operation for varying cells' SoC within a serial connection. Data are reported to the "Energy Management System" (EMS) of the BESS for power flow control within battery limits and allows the execution of operational strategies [16]. Batteries need a BMS in order to control how cells are charged and discharged. This BMS consists of a simple charge controller or a complex algorithm monitoring many variables [13]. BESS requires a BMS in order to monitor, maintain safe and optimal operation of each battery pack and for systems that have multiple sets of batteries that can operate independently, a System Supervisory Control (SSC) is for monitoring and control of the whole system.

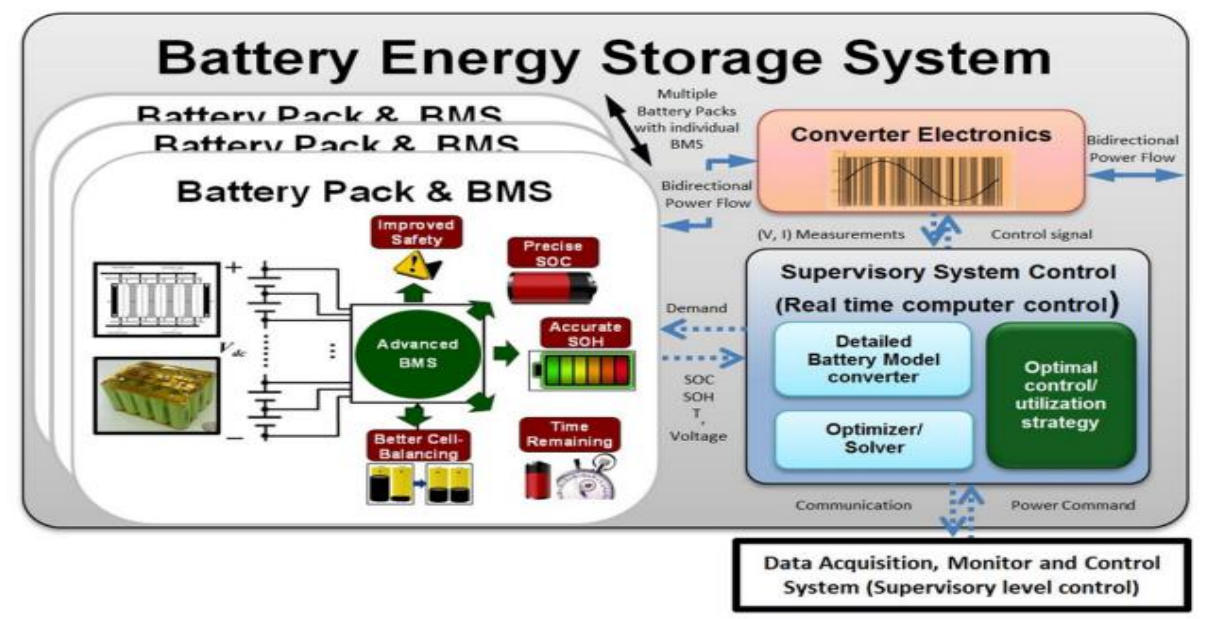

Figure 4. Schematic diagram for the implementation of a battery pack with BMS in the BESS [13]

As shown in Figure 4, the BMS and SCC can support the BESS with an optimal performance by providing optimal charging patterns, minimizing temperature across the system, balancing and, protecting the cells from internal degradation, etc. BESS can be controlled by the BMS and SCC by manipulating only current or voltage, and temperature (if cooling or heating system is available).

In large power systems, many battery packs are combined with individual BMS to create a high BESS capacity. In BESS, SSC is considered as an interface between the electrical network and the BMS. The data and information about battery packs are transmitted from BMS to SSC. When power is solicited by the grid, an optimal protocol is chosen by the SSC while accounting both grid demand and the state of the batteries. The power is called from individual packs by this SSC protocol in order to match with power demand [13].The energy of the battery can be calculated as in the equation (7) [17]:

$$
B_{b a t}=B_{b a t .0}+\int_{0}^{t} V_{b a t} I_{b a t} d t
$$

Where $B_{b a t . o}$ the initial battery charge, $V_{b a t}$ and $I_{b a t}$ are respectively the voltage and current of the battery. The accurate estimation of $S o C$ is necessary to calculate the correct value of energy of the battery. Also, the $S o C$ of the battery varies and it depends on time, it is expressed by the equation:

$$
\frac{\operatorname{SOC}(t)}{\operatorname{SOC}(t-1)}=\int_{T-1}^{T} \frac{P_{b a t}(t) \eta_{\text {bat }}}{V_{\text {bus }}} d t
$$

Where $V_{b u s}$ is the bus voltage in a micro-grid, $\eta_{b a t}$ indicates round-trip efficiency of the battery. The supplied power by the battery is symbolized by $P_{b a t}(t)$. Such round-trip efficiency is expressed as in equation (9): 


$$
\eta_{b a t}=\sqrt{\eta_{b a t}^{c}+\eta_{b a t}^{d}}
$$

Where $\eta_{b a t}^{c}$ and $\eta_{b a t}^{d}$ are respectively the charging and discharging efficiencies of the battery, respectively. The maximum value of SoC is noted by $S_{o} C_{\max }$ which is equal to the total capacity of the battery bank $\mathrm{C}_{\mathrm{n}}(\mathrm{Ah})$. It is expressed as following:

$$
C_{n}(A h)=\frac{N_{\text {bat }}}{N_{\text {bat }}^{s}} C_{b}(A h)
$$

Where $N_{b a t}$ is the total number of batteries, $\mathrm{C}_{\mathrm{b}}(\mathrm{Ah})$ represents the capacity of a single battery, $N_{\text {bat }}^{S}$ is the number of batteries which are connected in series. The minimum discharging value of a battery bank is denoted as $S o C_{\min }$. This minimum limit depends on the type of the battery and its usage in the BESS. The batteries can be connected in series to obtain the desired bus voltage. The number of these batteries which are connected in series to attain the required voltage is calculated as:

$$
N_{\text {bat }}^{S}=\frac{V_{\text {bus }}}{V_{\text {bat }}}
$$

Where $V_{\text {bat }}$ is the nominal voltage of a single battery. However, the maximum value of charging/discharging power of a battery at any time is expressed as in the equation (12):

$$
P_{b a t}^{\max }=\frac{N_{\text {bat }} V_{\text {bat }} I_{\text {bat }}^{\max }}{1000}
$$

Where $I_{\text {bat }}^{\max }$ represents the maximum charging current [17].

To determine the optimal operation of batteries systems in terms of cost, we firstly need to have a model that describes the total efficiency of an individual battery system by taking into consideration both the batteries and inverter losses. The internal resistance $\mathrm{R}_{\text {bat }}$ is the main parameter that impacts on the battery efficiency $\eta_{\text {bat }}$ [18]. By using the Thevenin equivalent circuit, the battery power $\mathrm{P}_{\text {bat }}$ is:

$$
P_{b a t}=V_{o c} I_{b a t}-R_{b a t} I_{b a t}^{2}
$$

Where $V_{o c}$ is the open-circuit-voltage (OCV). The battery efficiency for discharging current $I_{b a t}>0$ is

$$
\eta_{\text {bat_dis }}=\frac{\boldsymbol{P}_{\text {bat }}}{\boldsymbol{P}_{\text {cell }}}=\frac{\boldsymbol{V}_{\text {oc }} \boldsymbol{I}_{\text {bat }}-\boldsymbol{R}_{\text {bat }} I_{\text {bat }}^{2}}{\boldsymbol{V}_{\text {oc }} \boldsymbol{I}_{\text {bat }}}=1-\frac{\boldsymbol{R}_{\text {bat }} \boldsymbol{I}_{\text {bat }}}{\boldsymbol{V}_{\text {oc }}}
$$

Where the internal cell power $P_{c e l l}$ is the input power. The battery efficiency for charging current $I_{b a t}<0$ is

$$
\eta_{\text {bat_ch }}=\frac{\boldsymbol{P}_{\text {cell }}}{\boldsymbol{P}_{\text {bat }}}=1+\frac{\boldsymbol{R}_{\text {bat }} I_{\text {bat }}}{V_{\text {oc }}-\boldsymbol{R}_{\text {bat }} I_{\text {bat }}}
$$

For $\mathrm{V}_{\mathrm{oc}} \gg R I_{\text {bat }}$, we can approximate $\eta_{\text {bat_ch }}$ as follows:

$$
\eta_{\text {bat_ch }}=\eta_{\text {bat_dis }}=1-\left|\frac{\boldsymbol{R}_{\text {bat }} \boldsymbol{I}_{\text {bat }}}{\boldsymbol{V}_{\text {oc }}}\right|
$$

The battery efficiency is expressed as a function of $P_{b a t}$ by solving (12) for $I_{b a t}$ and substituting the resulting expression into (15) [18]:

$$
\boldsymbol{\eta}_{b a t}=1-\left|\frac{V_{o c}-\sqrt{V_{o c}^{2}-4 R_{b a t} P_{b a t}}}{2 V_{o c}}\right|
$$

A battery is represented by two important parameters which are the terminal voltage and the state-of-charge (SoC) as:

$$
\begin{gathered}
V_{b a t}=V_{o c}+R_{b a t} I_{b a t}-\frac{K Q_{b a t}}{Q_{b a t}+\int I_{b a t} d t}+A_{b a t} \exp \left(B_{b a t} \int I_{b a t} d t\right) \\
S o C=100\left(1+\frac{\int I_{b a t} d t}{Q_{b a t}}\right)
\end{gathered}
$$


Where $R_{b a t}$ is the battery internal resistance, $V_{o c}$ is the open-circuit voltage of the battery, $I_{b a t}$ is the charging current of the battery, $K$ is the polarization voltage, $Q_{b a t}$ is the battery capacity, $A_{b a t}$ is the exponential voltage, and $B_{b a t}$ is the exponential capacity. Charging or discharging of the battery can be ensured by controlling the bidirectional dc-dc converter.

$$
-\frac{P_{\text {Bat_N }}}{V_{\text {Bat }}} \leq I_{\text {bat }} \leq \frac{P_{\text {Bat_N }}}{V_{\text {Bat }}}
$$

Where $P_{B a t \_N}$ is the nominal power of the battery; $V_{B a t}$ is the rated voltage.

\section{MICRO-GRID MODEL DESCRIPTION}

\subsection{Case study}

In this example we present the operation of a simplified model of a micro-grid. The concerned microgrid is an electrical grid with a single-phase of $230 \mathrm{~V}$ AC where photovoltaic panels (PV) and wind turbine generator (WTG) with maximum power generation of $5 \mathrm{~kW}$ and $10 \mathrm{~kW}$ respectively. The main power sources are: grid utility, PV and WTG. The BESS with power capacity of $8 \mathrm{~kW}$ is controlled by a control system, it absorbs energy if there is an excess or it provides energy if there is a power deficiency in the micro-grid. Three ordinary houses consume a maximum power of $15 \mathrm{~kW}$ each are considered as loads. This micro-grid is connected to the utility grid through a pole-mounted power transformer. This transformer (primary $6.6 \mathrm{kV} / \mathrm{secondary} 230 \mathrm{~V}$ ) changes the AC voltage from three-phase $6.6 \mathrm{kV}$ of the utility grid into a single-phase $230 \mathrm{~V}$ on the micro-grid side. The frequency is $50 \mathrm{~Hz}$. The PV generation with batteries is DC power sources which require DC-AC converters to ensure connection to the power grid, thus, to be able to exchange energy. The initial SoC (State of Charge) of BESS is fixed at a constant value of $90 \%$. When there is power deficiency in the micro-grid, the utility grid compensates the missing power. If there is a power excess in the micro-grid, it will be transferred to the utility power system after the battery is being charged. Also, a comparative study is done to compare the response for different types of batteries used to support the electrical network during power fluctuations, thus, to take a decision about the battery type which is more suitable for power systems and particularly for energy management in micro-grids.

The micro-grid model is designed in RT-LAB platform with Matlab/Simulink and presented in Lab-view in order to show the behaviour and change in parameters of the micro-grid components (solar irradiance, wind speed, load consumption, charging/discharging power of the battery, etc.) by using real time simulation as it is presented in the Figure 5.

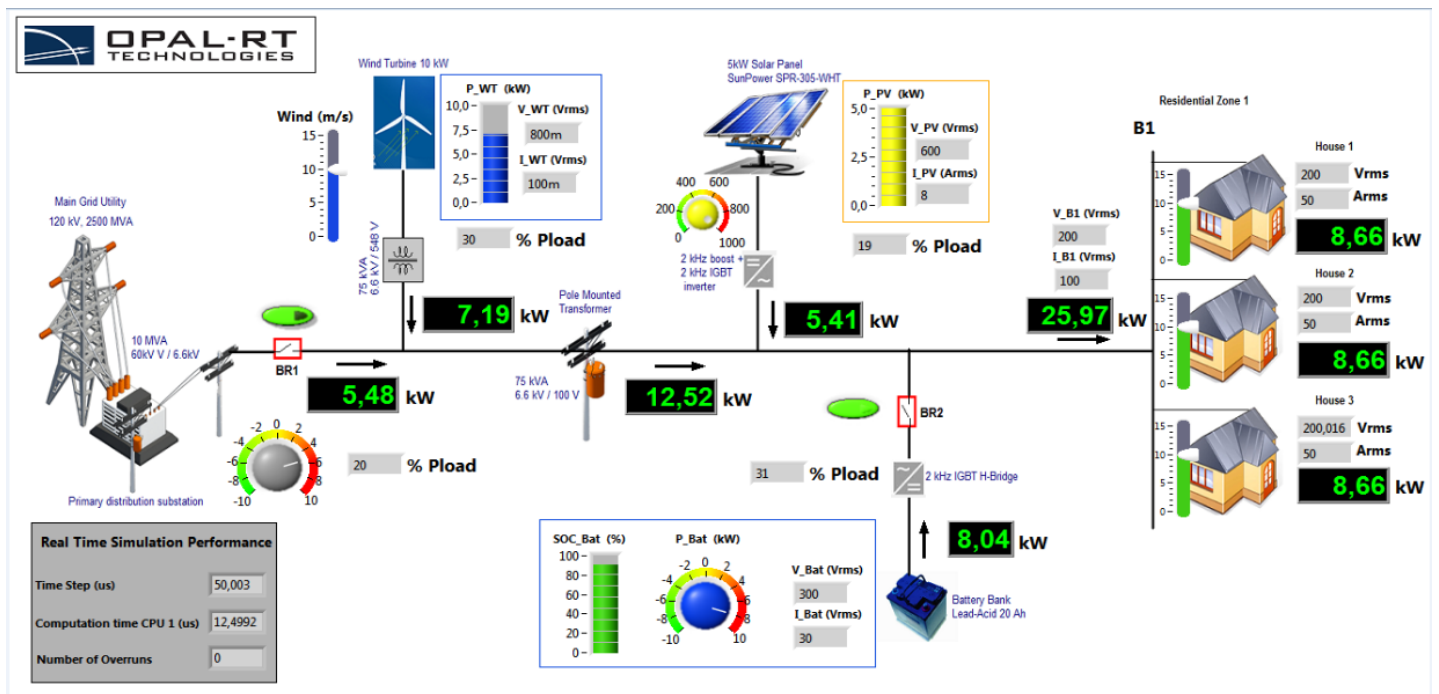

Figure 5. Micro-grid RT-LAB model representation in Labview

\subsection{Battery technologies}

Nowadays, different battery technologies are used for energy storage in power electrical systems requiring regulation, and energy management by providing or absorbing energy to/from the electrical grid.

\subsubsection{Lead-Acid batteries}


Among different types of battery technologies, Lead-Acid (LA) batteries are the most used for the electrical system applications. These batteries are well known in industrial and automotive applications, and they are effective in utility storage systems. Lead-acid batteries are economical energy storage devices, but they have a short life cycle. In general, these batteries need to be replaced every 4-5 years, which is considered as a constraint for the system life-time cost [17].

\subsubsection{Lithium-Ion batteries}

Recently, Lithium-ion (Li-ion) batteries are used more and more in electrical applications due to their high safety, volumetric and gravimetric energy densities characteristics, and lower maintenance. All these features make them to be light with smaller size than those of Lead-acid batteries. Also, the price of Li-ion batteries technology is decreasing at $8-16 \%$ every year [19].

\subsubsection{Nickel-Cadmium batteries}

Nickel-Cadmium (NiCd) batteries are well recognized by their high energy density and better life-span than lead-acid batteries. On the other hand, theses batteries are characterized with high values of self-discharge, and a memory effect which restricts their capacity according to battery usage. In addition, the composition of $\mathrm{NiCd}$ batteries is the extremely toxic Cadmium, which is considered as one of their disadvantages [20].

Thus, Li-ion batteries appears as a reliable solution which takes into account its high energy density better than lead-acid, NiCd and other type batteries, as well as their higher efficiency. Additionally, by using Li-ion batteries all the issues which are related to memory effect and toxic composition are avoided. Therefore, Liion batteries are usually more expensive than the other types of batteries despite of their prone to self-discharge [20]. Actually, lithium batteries have almost replaced the lead-acid batteries. Also, lithium-ion batteries have better environmental impact and a longer cycle life than lead-acid ones [21]. In fact, due to the integration of BMS, installations based on Li-ion batteries can be easily expanded in order to increase their capacity. On the other hand, regarding to the configuration, lead-acid batteries require more attention. Also, new lead-acid cells should never be combined with old ones (the new cells are usually more solicited due to their lower internal resistance and will degrade quickly. Whereas, lead-acid batteries can be recycled, they are composed of more than $90 \%$ of a recycled material. However, recycling industries for Li-ion batteries still relatively nascent and the percentage of batteries to be recycled is limited because of their ceiling designs [22].The RT-LAB microgrid model is represented in Labview as it is shown in Figure 5

Table 1. Characteristics of various batteries

\begin{tabular}{lccc}
\hline \multirow{2}{*}{ Battery parameters } & & Battery types \\
\cline { 2 - 4 } & Lead-Acid & Lithium-Ion & Nickel-Cadmium \\
\hline Nominal voltage (Volt) & $200 * \sqrt{2}$ & $200 * \sqrt{2}$ & $200 * \sqrt{2}$ \\
Rated capacity (Ah) & 1000 & 1000 & 900 \\
Initial State-Of-Charge (\%) & 90 & 90 & 1000 \\
Maximum capacity (Ah) & 1041.6667 & 329.2253 & 323.6214 \\
Fully charged voltage (Volt) & 307.9636 & 434.7826 & 200 \\
Nominal Discharge Current (A) & 200 & 0.0028284 & 0.0028284 \\
Internal Resistance (Ohms) & 0.0028284 & {$[301.8-279.5]$} & 5 \\
Exponential Zone [Voltage (volt), Capacity (Ah)] & {$[287.9-3.3]$} & {$[305.5-49.1]$} & 5 \\
Battery Response Time (seconds) & 5 & 5 & 5 \\
\hline
\end{tabular}

\section{REAL-TIME SIMULATION}

Due to the variation in many external factors such as unpredictable changes of weather, renewable energy resources in electrical grids are often considered as intermittent sources which require complex and more reliable control systems to regulate the flow and to improve the quality of power, voltage and frequency. The greater complexity of such systems, the more tests are required, all with high accuracy than ever. In addition, most of existing research about renewable energies mainly focuses on off-line simulation which has to go through a long research period and low efficiency. Thus, real-time simulation enables researchers to study the effects of multiple scenarios in near real conditions without risk, prior to deployment in the field ${ }^{2}$.The main purpose of the real-time simulation is to test electrical equipments in conditions as close as possible to reality. In fact, the real-time digital simulator should reproduce as possible the real phenomena and the dynamic behaviour of the controlled power system. The advantages of real-time simulation are: i) gaining time during

\footnotetext{
${ }^{2}$ https://www.opal-rt.com/microgrid-overview/ Viewed: 19-févr-2020
} 
the testing process, ii) resolve problems and detect faults at an earlier stage during the design process, iii) reduce new device testing cost under real conditions with more test in the lab and less tests on-site.

The real-time digital simulation of a power system to be tested and controlled passes through three important steps such as : i) a modelling phase which consists of putting equations for the system, then ii) a phase of algorithmic specification (choice of sampling time, discretization parameters), and finally iii) a phase of realtime implantation or execution. On the other hand, an advanced platform RT-LAB (REAL-TIME LABORATORY) for real time digital simulation is developed by the Canadian company: OPAL-RT Technologies, where hardware in the loop (HIL) simulation and online parameter adjustment can be implemented, which can shorten the research period and has good economical efficiency. In addition, RT-LAB can connect physical equipments into the simulator to make the simulation as close as possible to the reality and to obtain more accurate results.

\subsection{Hardware with software architecture of RT-LAB platform}

The RT-LAB digital simulator used in SCAMRE laboratory is composed of two main parts: software architecture and hardware architecture.

\subsection{Software architecture}

RT-LAB allows designing and modelling of the system under Matlab/Simulink environment with respect to some rules, and performing automatic code generation, then a transfer of this Simulink model to the Field Programmable Gate Array (FPGA) for implementation. Although, to make the model suitable for the real-time operation mode in RT-LAB, the model built in MATLAB/Simulink environment needs some modifications [23],[24]. RT-LAB is characterized with its many target processors and can divide a complex model into simple subsystems, then it implements parallel operations in multi-processors in order to accelerate the computing speed, which is the objective of real-time simulation ${ }^{3}$ [24].

\subsection{Hardware architecture}

The hardware architecture which is available in SCAMRE laboratory is constituted of two simulators which are connected to each other; WANDA-4u and the OP-5600. The target is composed of two CPU processors (Intel Xenon six-core, 3.33GHz, 6.4GT/s), including 2 activated cores and $16 \mathrm{I} / \mathrm{O}$ for Wanda and two CPU processors (Xenon six-core, 3.46GHz, 6.4GT/s) including 2 activated cores with 16 I/O for OP5600. The execution of different models is the main task of the target. As it is shown in Figure 6, the role of the host computer is the development, editing, verification and compilation of models. It is also considered as a console or command station to ensure the control and observation during simulation process.

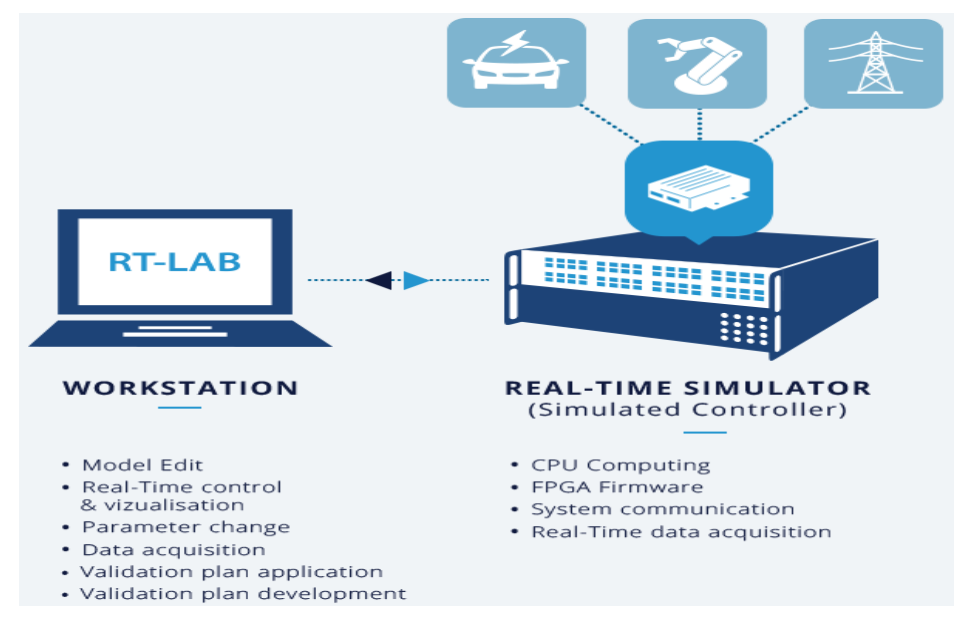

Figure 6. Topology of hardware architecture in RT-LAB platform

\section{RESULTS AND ANALYSIS}

The real time digital simulation verifies the reliability, feasibility and efficiency of the control system by using RT-LAB platform consists of testing the response of energy storage batteries during and after power

\footnotetext{
${ }^{3}$ https://www.opal-rt.com/opal tutorial/preparing-simulink-model-real-time-execution/ viewed: 19-févr-
} 2020 
fluctuations in the micro-grid. Also, comparison of energy storage system effectiveness during such critical situation between three types of batteries which are connected to the micro-grid is conducted through the following cases: Case 1: Only Lead-Acid batteries are connected to the micro-grid; Case 2: Only Lithium-Ion batteries are connected to the micro-grid; Case 3: Only Nickel-Cadmium batteries are connected to the microgrid. The characteristics of these types of batteries are given in the Table 1.

The simulation results are presented in the following figures for each case:

Figure 7 (a)- Lead-Acid batteries response during power fluctuations in the micro-grid;

Figure 7 (b)- Supplied active power from the main utility grid while only Lead-Acid batteries are connected to the micro-grid;

Figure 7 (c)- Lithium-Ion batteries response during power fluctuations in the micro-grid;

Figure 7 (d)- Supplied active power from the main utility grid while Lithium-Ion batteries are connected to the micro-grid;

Figure 7 (e)- Nickel-Cadmium batteries response during power fluctuations in the micro-grid;

Figure 7 (f)- Supplied active power from the main utility grid while only Nickel-Cadmium batteries are connected to the micro-grid

When only the Lead-Acid batteries which are connected to the micro-grid, the response time of the BESS for the active power fluctuations in the micro-grid is around 40 seconds. The power of Lead-Acid batteries $P_{b a t}$ fluctuates from 0 seconds to 40 seconds as it is drawn in Figure 7 (a), also, from 0 seconds to 40 seconds, the active power $P_{\text {sec }}$ supplied or absorbed by the utility main grid fluctuates but after 40 seconds it stabilizes as it is shown in Figure 7 (b).

In the case of Lithium-ion batteries, the response time of the BESS for the active power fluctuations in the micro-grid is around 8 seconds, the power of Lithium-Ion batteries $P_{b a t}$ fluctuates from 0 seconds to 8 seconds in order to support the micro-grid by delivering or absorbing energy as it is drawn in Figure 7 (c), but after 8 seconds $P_{b a t}$ stabilizes. From 0 seconds to 8 seconds, the active power $P_{s e c}$ supplied or delivered by the utility main grid fluctuates but after 8 seconds it stabilizes as it is shown in Figure 7 (d).

For the Nickel-Cadmium batteries, the active power fluctuation in the micro-grid is during 38 seconds. The power of Nickel-Cadmium batteries $P_{b a t}$ fluctuates from 0 seconds to 38 seconds in order to support the micro-grid by delivering or absorbing energy as it is drawn in Figure 7(e), but after 38 seconds it stabilizes. From 0 seconds to 38 seconds, the active power $P_{\text {sec }}$ supplied or absorbed by the utility grid fluctuates but after 38 seconds it stabilizes as it is shown in Figure 7 (f).

For every type of battery technology, the simulation results show that the Lithium-ion batteries have contributed for the reduction of active power fluctuation in 7 to 8 seconds faster than lead-Acid and NickelCadmium batteries which have responded in 38 seconds and 40 seconds respectively. Such difference in response time between the three types of batteries is due to the difference in their material characteristics as well as impedance parameters which impact on the efficiency and performance of each type of battery. In addition, the active power supplied or absorbed from the batteries $P_{b a t}$ has the same variation as the power supplied or absorbed by the utility main grid $P_{s e c}$ which confirms that BESS compensate the missing power or absorb the excess of power in the micro-grid. Also, BESS can provide active and reactive power, thus, it can regulate frequency and voltage. The fast dynamic response of a BESS can support frequency and voltage regulation process in grid-connected mode during load/power fluctuations or any disturbance/fault occurrence because frequency depends on active power variation, and voltage depends on reactive power variaion; these results confirms that the BESS has really contributed to support the micro-grid by supplying or absorbing energy in order to reduce active power fluctuation and electrical grid instability. As the Lithium-Ion batteries have contributed for system stability faster than the Lead-Acid and Nickel-Cadmium, such result proves that Li-ion is an efficient solution to support micro-grids in terms of frequency regulation, reduction of power fluctuation, peak shaving, etc. If a BESS delivers power as quickly as possible, it means that it delivers more quantity of energy in a short time, which is a desirable behaviour that contributes to improve power grid stability.

IJEEI, Vol.9, No. 2, June 2021: 371 - 383 


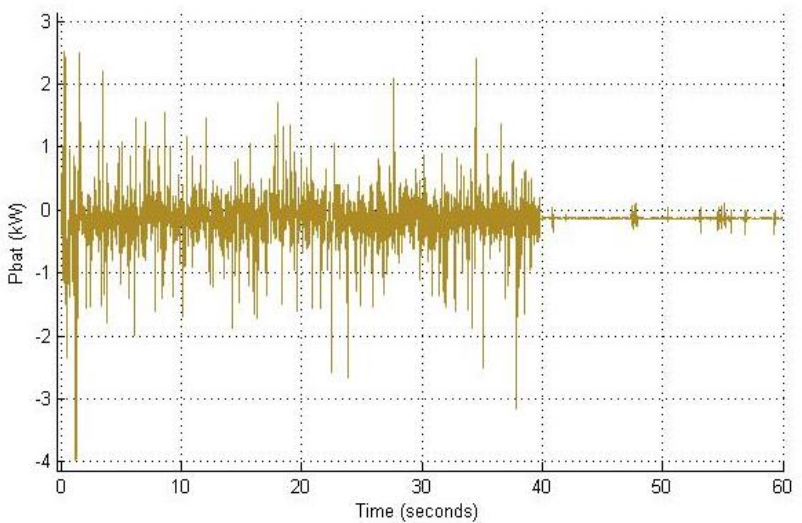

(a)

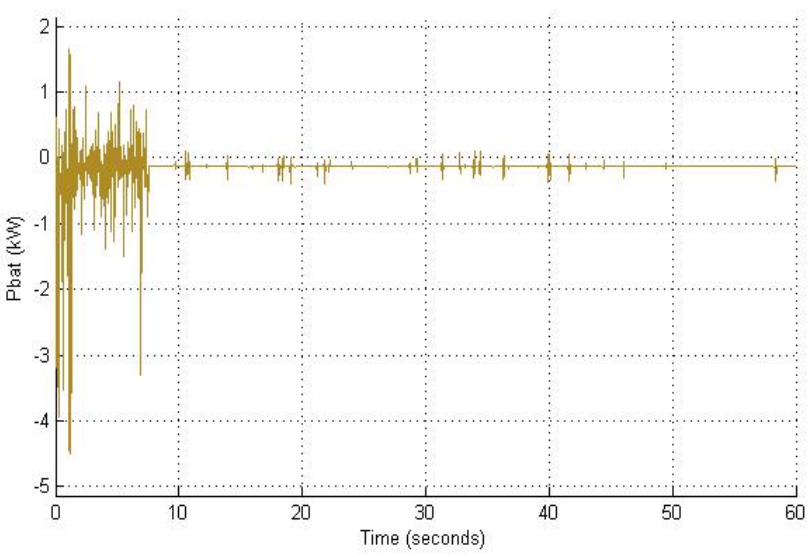

(c)

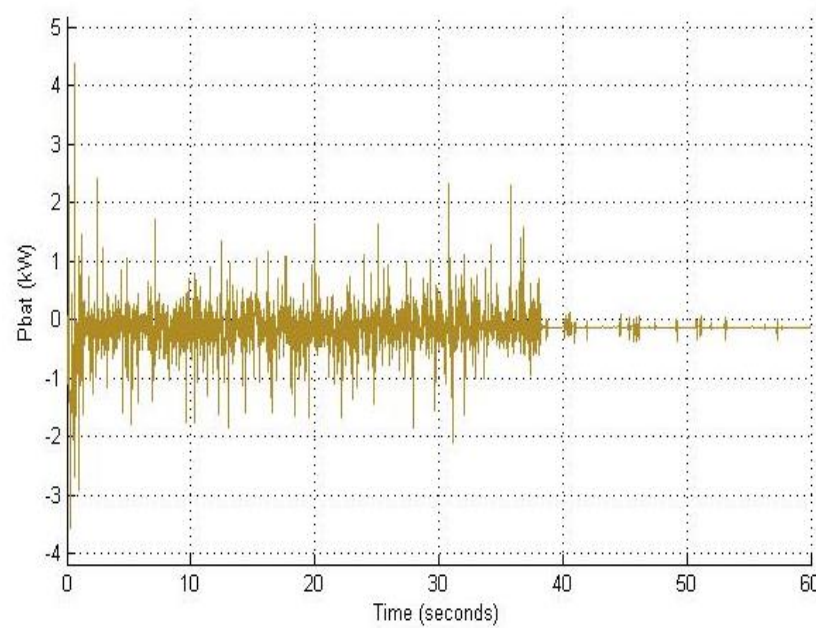

(e)

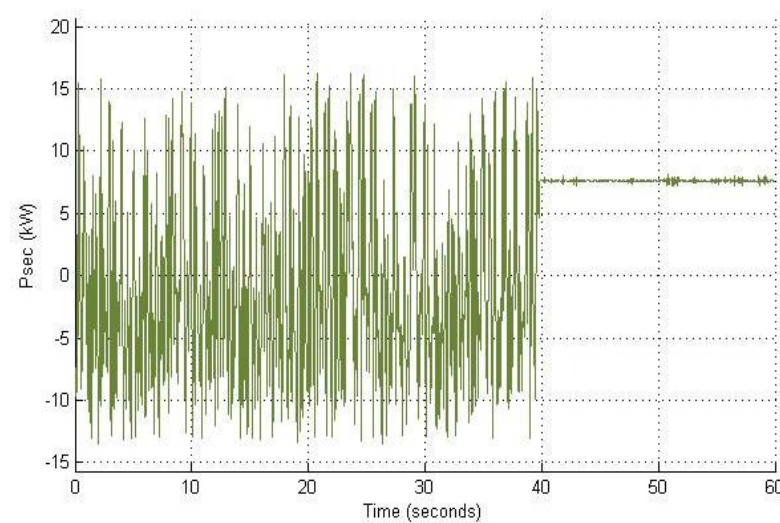

(b)

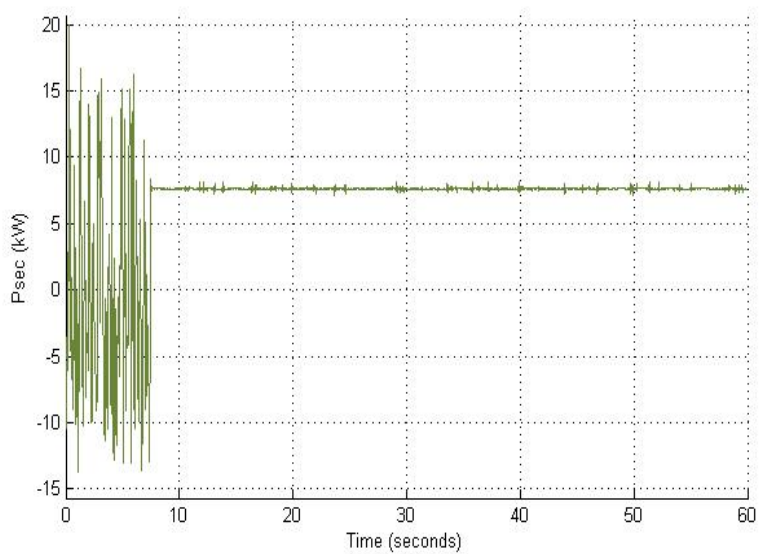

(d)

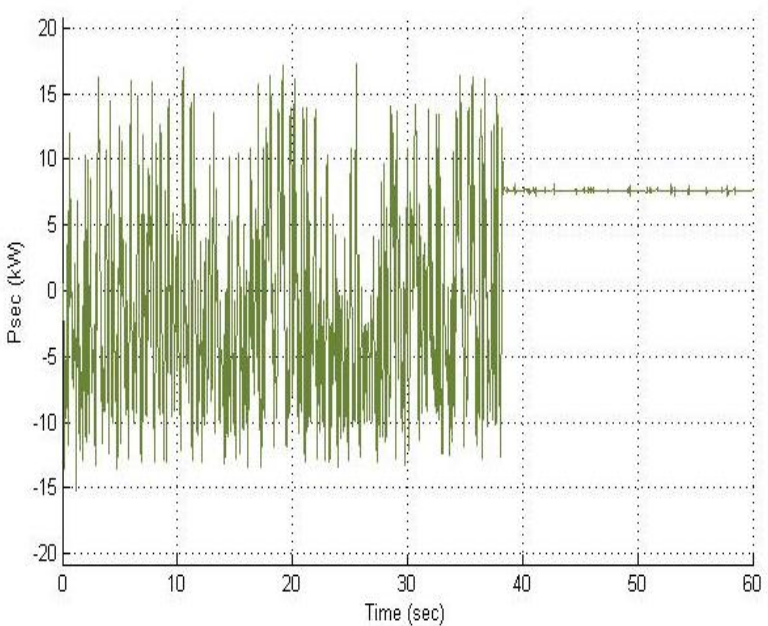

(f)

Figure 7. Results of various simulations- BESS and utility electrical grid response 


\section{CONCLUSION AND FUTURE WORK}

In this article, we are interested in the verification of the behaviour of BESS and how it manages its state-of-charge (SoC), therefore, ensuring that its available energy can support the micro-grid during disturbances such power fluctuations and how quickly BESS can deliver or absorb power as a response to any undesirable phenomena in order to guarantee the electrical grid stability and power balance. The real-time simulation based on RT-LAB platform of SCAMRE laboratory helped us to study different scenarios in very close to real conditions, and to make several tests on the models conceived without risk, before deploying them in the field. The simulation results show that BESS can effectively support micro-grids during perturbations such as power fluctuations. Based on the real time digital simulation results, it is confirmed that the battery energy storage system is an efficient solution for the reduction of power fluctuations in micro-grids due to its fast response and as its location near the loads allows the compensation of the loss in the distribution grid.

In addition, The Lithium-Ion batteries are the most suitable solution among other types of batteries for micro-grid operation and stability due to their technical benefits for power system in terms of safety, reliability flexibility, and its fast response.

As a future perspective, in order to confirm about the efficiency of energy storage system on microgrid operation, the proposed work will be implemented as experimentation. A comparison between experimental and the real time simulation results will be adopted. Such comparison will be an important approach for the development of BESS utilization in power system with high rate, more efficiency and less cost.

\section{REFERENCES}

[1] F. Conteh, S. Tobaru, H. O. R. Howlader, A. Yona, et T. Senjyu, «Energy management systems for hybrid distributed generation sources in grid connected and stand-alone micro-grids », Journal of Renewable and Sustainable Energy, vol. 9, nº 6, p. 065301, nov. 2017, doi: 10.1063/1.4998773.

[2] M. A. Hannan, S. Y. Tan, A. Q. Al-Shetwi, K. P. Jern, et R. A. Begum, « Optimized controller for renewable energy sources integration into microgrid: Functions, constraints and suggestions », Journal of Cleaner Production, vol. 256, p. 120419, mai 2020, doi: 10.1016/j.jclepro.2020.120419.

[3] M. Cheng, S. S. Sami, et J. Wu, « Virtual Energy Storage System for Smart Grids », Energy Procedia, vol. 88, p. 436-442, juin 2016, doi: 10.1016/j.egypro.2016.06.021.

[4] A. Aktas, K. Erhan, S. Ozdemir, et E. Ozdemir, « Experimental investigation of a new smart energy management algorithm for a hybrid energy storage system in smart grid applications », Electric Power Systems Research, vol. 144, p. 185-196, mars 2017, doi: 10.1016/j.epsr.2016.11.022.

[5] W. Wang, S. Dmitry, et L. Sun, « Discharging scheme for distributed battery storage devices in smart grid », in 2016 Australasian Universities Power Engineering Conference (AUPEC), Brisbane, Australia, sept. 2016, p. 1-5, doi: 10.1109/AUPEC.2016.7749288.

[6] M. LEGRAIVE, « Realisation of Lithium battery model for microgrid applications and Validation with Real-time Simulation Platform », Université catholique de Louvain, Ecole polytechnique de Louvain, Louvain-la-Neuve, Belgium, 2016.

[7] Jin-Hong Jeon et al., « Development of Hardware In-the-Loop Simulation System for Testing Operation and Control Functions of Microgrid», IEEE Trans. Power Electron., vol. 25, no 12, p. 2919-2929, déc. 2010, doi: 10.1109/TPEL.2010.2078518.

[8] Y. Yang, S. Bremner, C. Menictas, et M. Kay, « Battery energy storage system size determination in renewable energy systems: A review », Renewable and Sustainable Energy Reviews, vol. 91, p. 109-125, août 2018, doi: 10.1016/j.rser.2018.03.047.

[9] C. F. Silva, « A Model of a Battery Energy Storage System for Power Systems Stability Studies », p. 8.

[10] N. L. Diaz, T. Dragicevic, J. C. Vasquez, et J. M. Guerrero, « Intelligent Distributed Generation and Storage Units for DC Microgrids-A New Concept on Cooperative Control Without Communications Beyond Droop Control », IEEE Trans. Smart Grid, vol. 5, no 5, p. 2476-2485, sept. 2014, doi: 10.1109/TSG.2014.2341740.

[11] D. Fendri et M. Chaabene, «Dynamic model to follow the state of charge of a lead-acid battery connected to photovoltaic panel», Energy Conversion and Management, vol. 64, p. 587-593, déc. 2012, doi: 10.1016/j.enconman.2012.05.027.

[12] Jianwei Wang, Xisheng Tang, et Zhenggang Yin, « Research on medium voltage battery energy storage system based on RT-LAB », in 2014 IEEE Conference and Expo Transportation Electrification Asia-Pacific (ITEC AsiaPacific), Beijing, China, août 2014, p. 1-5, doi: 10.1109/ITEC-AP.2014.6940996.

[13] M. T. Lawder, « Modeling, Simulation, and Analysis of Lithium-Ion Batteries for Grid-Scale Applications », p. 233.

[14] K. S. Sandhu et A. Mahesh, «A new approach of sizing battery energy storage system for smoothing the power fluctuations of a PV/wind hybrid system », International Journal of Energy Research, vol. 40, nº 9, p. 1221-1234, 2016, doi: 10.1002/er.3511.

[15] Q. Liao, B. Sun, Y. Liu, J. Sun, et G. Zhou, « A techno-economic analysis on NaS battery energy storage system supporting peak shaving », International Journal of Energy Research, vol. 40, $\mathrm{n}^{\circ}$ 2, p. 241-247, 2016, doi: 10.1002/er.3460. 
[16] H. Hesse, M. Schimpe, D. Kucevic, et A. Jossen, «Lithium-Ion Battery Storage for the Grid-A Review of Stationary Battery Storage System Design Tailored for Applications in Modern Power Grids », Energies, vol. 10, no 12, p. 2107, déc. 2017, doi: 10.3390/en10122107.

[17] S. Dhundhara, Y. P. Verma, et A. Williams, « Techno-economic analysis of the lithium-ion and lead-acid battery in microgrid systems ", Energy Conversion and Management, vol. 177, p. 122-142, déc. 2018, doi: 10.1016/j.enconman.2018.09.030.

[18] P. Fortenbacher, J. L. Mathieu, et G. Andersson, « Modeling and Optimal Operation of Distributed Battery Storage in Low Voltage Grids », IEEE Transactions on Power Systems, vol. 32, n 6, p. 4340-4350, nov. 2017, doi: 10.1109/TPWRS.2017.2682339.

[19] S. X. Chen, K. J. Tseng, et S. S. Choi, « Modeling of Lithium-Ion Battery for Energy Storage System Simulation », in 2009 Asia-Pacific Power and Energy Engineering Conference, Wuhan, China, mars 2009, p. 1-4, doi: 10.1109/APPEEC.2009.4918501.

[20] V. Vega-Garita, A. Hanif, N. Narayan, L. Ramirez-Elizondo, et P. Bauer, « Selecting a suitable battery technology for the photovoltaic battery integrated module », Journal of Power Sources, vol. 438, p. 227011, oct. 2019, doi: 10.1016/j.jpowsour.2019.227011.

[21] «Online Identification of Thevenin Equivalent Circuit Model Parameters and Estimation State of Charge of Lithium-Ion Batteries - IEEE Conference Publication ». https://ieeexplore.ieee.org/abstract/document/8493924 (consulté le févr. 18, 2020).

[22] E. Lockhart et al., « Comparative Study of Techno-Economics of Lithium-Ion and Lead-Acid Batteries in MicroGrids in Sub-Saharan Africa », National Renewable Energy Lab. (NREL), Golden, CO (United States), NREL/TP7A40-73238, juin 2019. doi: 10.2172/1526204.

[23] A. Idir et M. Kidouche, «RT-LAB AND DSPACE: TWO SOFTWARES FOR REAL TIME CONTROL OF INDUCTION MOTORS », p. 10.

[24] Y. Zhao, L. Shi, et L. Yao, « Real-time simulation on wind power system dynamics incorporating STATCOM », in 2013 IEEE PES Asia-Pacific Power and Energy Engineering Conference (APPEEC), Kowloon, Hong Kong, déc. 2013, p. 1-6, doi: 10.1109/APPEEC.2013.6837210. 